\title{
Application of the Variational Mode Decomposition for Power Quality Analysis
}

\author{
Kewei Cai, ${ }^{1,2}$ Wenping Cao, ${ }^{2}$ Zheng Liu, ${ }^{3 *}$ Wei Wang, ${ }^{1}$ Guofeng ii $^{3}$
}

${ }^{1}$ College of Information Engineering, Dalian Ocean University, Dalian, 116023, China

caikw0602@live.cn (K. C.); ww_wangwei@dlou.edu.cn (W. W)

2 School of Engineering and Applied Science, Aston University, Birmingham, B4 7ET, UK

w.p.cao@aston.ac.uk (W.C.)

3 School of Electrical Engineering, Dalian University of Technology, Dalian, 116023, China zhengliushef@163.com (Z.L.); guofenli@dlut.edu.cn (G. L.)

*Correspondence: zhengliushef@163.com

\begin{abstract}
Harmonics and interharmonics in power systems distort the grid voltage, deteriorate the quality and stability of the power grid. Therefore, rapid and accurate harmonic separation from the grid voltage is crucial to power system. In this paper, a variational mode decomposition (VMD)based method is proposed to separate harmonics and interharmonics in the grid voltage. The method decomposes the voltage signal into fundamental, harmonic, interharmonic components through the frequency spectrum. An empirical mode decomposition (EMD) and an ensemble empirical mode decomposition (EEMD) can be combined with the independent component analysis (ICA) to analyse the harmonics and intherharmonics. By comparing EMD-ICA, EEMD-ICA methods, the proposed method has several advantages: 1) a higher correlation coefficient $R$ of all the components is found; 2) it requires much less time to accomplish signal separation; 3) amplitude, frequency and phase angle are all retained by this method. The results obtained from both synthetic and real-life signals demonstrate the good performance of the proposed method.
\end{abstract}

Keywords-EEMD-ICA, interharmonics, power decomposition (VMD).

\section{INTRODUCTION}

$\mathrm{I}^{\mathrm{n}}$ $\mathrm{n}$ recent years, concerns over the power quality with harmonics and interharmonics have been increasing since the wide use of nonlinear loads and high-switching power electronic devices in the industry process, such as electric arc furnaces, HVDC, grid-tied distributed generation [1-3]. These give rise to disturbances to the power grids, which greatly affect the safe and economical operations of power systems, decrease the lifetime and performance of electrical equipment [4]. Harmonic analysis, including detection and separation of harmonics and interharmonics, is an important task to provide an adequate solution to power system quality and stability problems. However, separation of interharmonics is difficult to achieve, since their frequencies are irregular and not multiples of the fundamental frequency. In this case, there is an increasing need for new approach to decompose both harmonics and interharmonics from the grid voltage.

In the literature, several methodologies have been proposed to identify different harmonics and interharmonics, such as the discrete fourier transform (DFT) [5], the wavelet analysis [6], and the blind source separation (BSS) [7]. Nowadays, BSS has been most widely used for harmonics separation from grid voltage, which is based on independent component analysis (ICA), due to its non-affected by frequency leakage and hurdle effect property [7]. However, the traditional ICA method needs that the number of observation recordings is more than original source, whereas grid voltage is single channel original source. Hence, harmonics and interharmonics separation of grid voltage belongs to single channel ICA problem, which could be addressed by the single channel independent component analysis (SCICA) algorithm [8]. The harmonic and interharmonic components from single channel grid voltage can be separated through constructing virtual channels of it based on SCICA [9, 10]. However, this method needs prior knowledge of voltage signal to construct the channels. Moreover, the number of virtual channel signals requires twice as much as the number of harmonic and interharmonic frequencies. Therefore, the complexity and efficiency of this method would be influenced seriously by the number of signal frequencies. The eigen value decomposition (EVD)-FastICA and the least square method (LSM)-FastICA methods [11, 12] are also used to separate harmonics in grid voltage. These two methods can separate harmonic and interharmonic with good performance, whereas also need the prior knowledge of voltage source frequency. In addition, the ICA algorithm combining with kurtosis and correlation coefficient can also extract the harmonics from grid voltage $[13,14]$. Nevertheless, the method only separates 3, 5, 7 order harmonics, whereas interharmonics are not included.

To overcome the drawbacks of SCICA, an empirical-mode decomposition (EMD) $[15,16]$ combined ICA (EMD-ICA) algorithm is proposed to extract harmonics from single channel source [17]. This method does not need any prior knowledge of original source and is widely used in single channel signal 
separation [18]. It is able to extract signal from high noise environment [19]. The research shows the good performance of the method by comparing with two other available algorithms, SCICA and wavelet independent component analysis (WICA) respectively. The principal component analysis (PCA) algorithm is introduced into the ensemble empirical-mode decomposition (EEMD)-ICA based method to reduce dimensionality of intrinsic mode functions (IMFs) and simplified algorithm [19]. Slope-EEMD (SEEMD) method has been proposed to overcome edge effect in EEMD algorithm [20-22]. In spite of advantages of EMD/EEMD based methods such as no need any prior knowledge of original signal, there are some severe limitations of it: 1) the different IMFs separated by EMD have mode mixing; 2) although, EEMD could make better effect on mode mixing, there are still some apparent limitations such as noise and sampling sensitivity, lack of mathematical theory etc. Therefore, the post-processing based ICA is required. The ICA could extract independent components from IMFs. In the meantime it would eliminate the amplitude of the original source (for example voltage amplitude) .

In this paper, a method was proposed to separate harmonics and interharmonics from grid voltage based on variational mode decomposition (VMD). This method is an entirely nonrecursive decomposition and can decompose multicomponent signal into a finite number of band-limited IMFs [23, 24]. The VMD algorithm is more robust to noise and sampling than EMD-based methods and has been used to extract signals in some fields such as biology [25], seismic [26] and acoustic [27]. However, harmonics and interharmonics from grid voltage by means of VMD algorithm is not reported. Synthetic and reallife signals were both used to validate the superiority and strength of the proposed method in this paper. The EEMD-ICA algorithm was also used to separate harmonic and interharmonic signals. The results of VMD method were compared to EMD/EEMD-ICA method to show the good performance of the algorithm in both simulation and real-life application.

\section{Methodologies}

VMD is a new, intrinsic and adaptive method. It is based on tikhonov regularization algorithm, wiener filter and hilbert transformation. The proposed method could estimate the corresponding modes with relevant bands adaptively and concurrently. This part illustrated the theory and solution of VMD method.

\section{A. Theory of $V M D$}

VMD decomposes a single channel multicomponent signal into an ensemble of band-limited IMFs, which are defined as an AM-FM signals with sparsity property. The IMF $u(t)$ is illustrated as:

$$
u(t)=A(t) \cos (\varphi(t))
$$

where the $\varphi(t)$ is phase, which is a nondecreasing function, and the instantaneous frequency $\omega(t)=\varphi^{\prime}(t)$ and $A(t)$ is the amplitude of the IMF.

1) The structure of VMD

The goal of VMD is to decompose a multicomponent signal into a finite number of sub-signals(modes), $u(k)$, that compact around a center frequency $\omega(k)$. The process of making VMD is listed as follows:

(1) Obtain the unilateral frequency spectrum of each mode through computing the associated analytic signal by means of Hilbert transform:

$$
\left(\delta(t)+\frac{j}{\pi t}\right) \times u_{k}(\mathrm{t})
$$

(2) Shift the frequency spectrum of each mode to baseband by multiplying an exponential tuned with estimated center frequency:

$$
\left[\left(\delta(t)+\frac{j}{\pi t}\right) \times u_{k}(\mathrm{t})\right] e^{-j \omega_{k} t}
$$

(3) Calculate the bandwidth of each mode by means of the squared $L^{2}$-norm of the gradient. The constrained variational problem is as follows:

$$
\begin{gathered}
\min _{\left\{u_{k}\right\},\left\{\omega_{k}\right\}}\left\{\left[\left(\delta(t)+\frac{j}{\pi t}\right) \times u_{k}(\mathrm{t})\right] e^{-j \omega_{k} t}\right\} \\
\text { s.t. } \sum u_{k}=f
\end{gathered}
$$

where $\left\{u_{k}\right\}=\left\{u_{1}, \cdots, u_{k}\right\}$ and $\left\{\omega_{k}\right\}=\left\{\omega_{1}, \cdots, \omega_{k}\right\}$ are all the modes with short bandwidth property and their center frequencies, respectively.

\section{2) The solution of VMD}

A quadratic penalty $\alpha$ and Lagrangian multipliers $\lambda$ are introduced to address (4) to make constrained problem to be unconstrained. The Lagrangian $L$ is illustrated as follows ${ }^{[22]}$ :

$$
\begin{array}{r}
L\left(\left\{u_{k}\right\},\left\{\omega_{k}\right\}, \lambda\right)=\alpha \sum_{k}\left\|\partial_{t}\left[\left(\delta(t)+\frac{j}{\pi t}\right) u_{k}(\mathrm{t})\right] e^{-j w_{k} t}\right\|_{2}^{2}+ \\
\left\|f(t)-\sum_{k} u_{k}(t)\right\|_{2}^{2}+\left\langle\lambda(t), f(t)-\sum_{k} u_{k}(t)\right\rangle
\end{array}
$$

In order to solve the variational problem in (5), alternate direction method of multipliers (ADMM) [28-30] is used to produce different decomposed modes and calculate the center frequencies. Each mode and center frequency obtained from solutions in spectral domain can be represented as:

$$
\begin{gathered}
\hat{u}_{k}^{n+1}(\omega)=\frac{\hat{f}(\omega)-\sum_{i \neq k} \widehat{u}_{i}(\omega)+(\widehat{\lambda}(\omega) / 2)}{1+2 \alpha\left(\omega-\omega_{k}\right)^{2}} \\
\omega_{k}^{n+1}=\frac{\int_{0}^{\infty} \omega\left|\widehat{u}_{k}(\omega)\right|^{2} d \omega}{\int_{0}^{\infty}\left|\widehat{u}_{k}(\omega)\right|^{2} d \omega}
\end{gathered}
$$

It is concluded that $\hat{u}_{k}^{n+1}(\omega)$ is the result of Wiener filter of the resident part $\hat{f}(\omega)-\sum_{i \neq k} \hat{u}_{i}(\omega) ; \omega_{k}^{n+1}$ is the center frequency [23]. The kth mode $u_{k}$ can be obtained by means of inverse Fourier Transform.

Therefore, the steps of VMD algorithm can be concluded as follows:

(1) Initialise the $\left\{\hat{u}_{k}^{1}=0\right\},\left\{\omega_{k}^{1}=0\right\},\left\{\hat{\lambda}^{1}=0\right\}$, and $n=0$;

(2) Update the $u_{k}$ and $\omega_{k}$ repeatedly according to (6), (7);

(3) Update dual ascent $\lambda$ according to:

$$
\hat{\lambda}^{n+1}=\hat{\lambda}^{n}+\tau\left(\hat{f}(\omega)-\sum_{k} \hat{u}_{k}^{n+1}(\omega)\right)
$$

(4) Repeat step (2), (3), until convergence: $\sum_{k}\left\|\hat{u}_{k}^{n+1}-\hat{u}_{k}^{n}\right\|_{2}^{2} /$ $\left\|\hat{u}_{k}^{n}\right\|_{2}^{2}<\varepsilon$.

Through the above process, a finite number IMFs $u_{k}$ with specific sparsity properties can be obtained by VMD nonrecursively. Actually, in this part, "finite number of IMFs" means that the input signal can be decomposed into (finite rather than infinite) number of IMFs. In this method, the specific sparsity properties refer to the central frequency of an IMF, where each mode has only one central frequency. The 


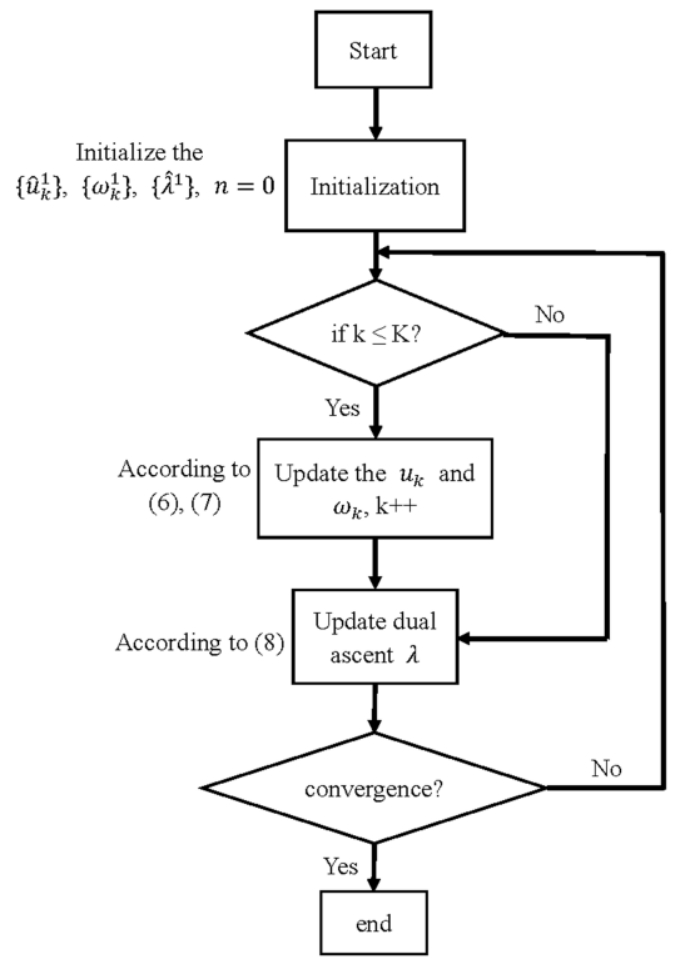

Fig. 1. Flow chart of the VMD

input signal is decomposed by the VMD method as per central frequency of harmonics and interharmonics. This algorithm is more robust to noise on account that the wiener filter is embedded in the modes update. A flow chart for VMD solution is shown in Fig. 1 [23], [31].

\section{B. Mathematical model of Grid Voltage}

Grid voltage signal was composed with 3 kinds of components, which are fundamental, harmonic and interharmonic components. The mathematical model is shown as follows:

$$
\begin{aligned}
v(t)= & A_{m} \sin (\omega t+\psi)+\sum H_{m_{\_} n_{1}} \sin \left(n_{1} \omega t+\varphi_{1}\right)+ \\
& \sum H_{\text {interm } \_} n_{2} \sin \left(n_{2} \omega t+\varphi_{2}\right)
\end{aligned}
$$

where, $\omega=2 \pi f, f=50 \mathrm{~Hz}, A_{m} \sin (\omega t+\psi)$ is fundamental component, $A_{m}, \psi$ are amplitude and initial phase of fundamental component respectively; $\sum H_{m_{-} n_{1}} \sin \left(n_{1} \omega t+\right.$ $\left.\varphi_{1}\right)$ is harmonics component, $H_{m \_n}, \varphi_{1}$ are amplitude and initial phase of harmonics component respectively, $n_{1}$ is number of harmonics, where $n_{1}=1,3,5, \ldots, 2 n+1, \quad(n$ is positive integer); $\sum H_{\text {interm } \_n} n_{2} \sin \left(n_{2} \omega t+\varphi_{2}\right)$ is interharmonics component, $H_{\text {interm } \_n_{2}}, \varphi_{2}$ are amplitude and initial phase of interharmonics component respectively, $n_{2}$ is frequency parameter, where $0<n_{2}<1$.

\section{PARAMETER SELECTION}

The selection of the key parameters for the VMD method is illustrated in this subsection.

\section{A. Lagrangian Multiplier $\lambda$}

The Lagrangian multiplier is selected to enforce the equality constraint of reconstruction. It can be set to zero if exact reconstruction is not required, particularly in noisy condition. In this paper, Lagrangian multiplier $\lambda$ is initialised to zero, furthermore, its update parameter $\tau=0$ in Eq. 8 [22] to make no update of $\lambda$.

\section{B. Number of Modes K:}

The number of modes $\mathrm{K}$ is one of the key parameters of VMD. It is crucial to be precisely chosen before VMD starts. Parameter K stipulates that how many modes can be separated through VMD. When $\mathrm{K}$ is small, there are not enough modes to be decomposed, hence, multi-modes components have to be in one mode, which named mode mixed. It would also lead instantaneous centre frequency to be vibrated instead of constant; whereas when $\mathrm{K}$ is large, much more modes have to be decomposed, it leads that one mode component would be mixed into different modes, which named mode redundant. It would increase computation stress and decrease system efficiency. In this paper, we choose $\mathrm{K}$ according to wave packages of FFT of voltage signal in the frequency domain in order to obtain an appropriate $\mathrm{K}$.

\section{Regularization factor $\alpha$ :}

The Eq. 5 is the classical Tikhonov regularization problem when $\lambda=0$ and the penalty parameter $\alpha$ is actually regularization factor. When $\alpha$ is small, it is easily to satisfy the constraint term $\left\|f(t)-\sum_{k=1}^{K} u_{k}(t)\right\|_{2}^{2}$, whereas the penalty

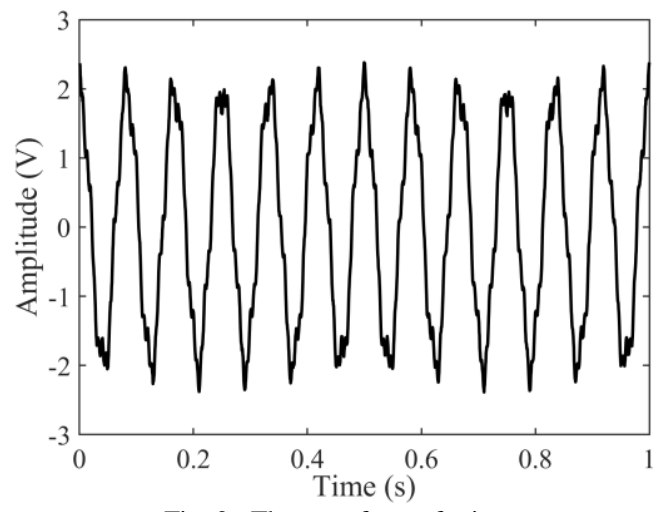

Fig. 2. The waveform of mixture

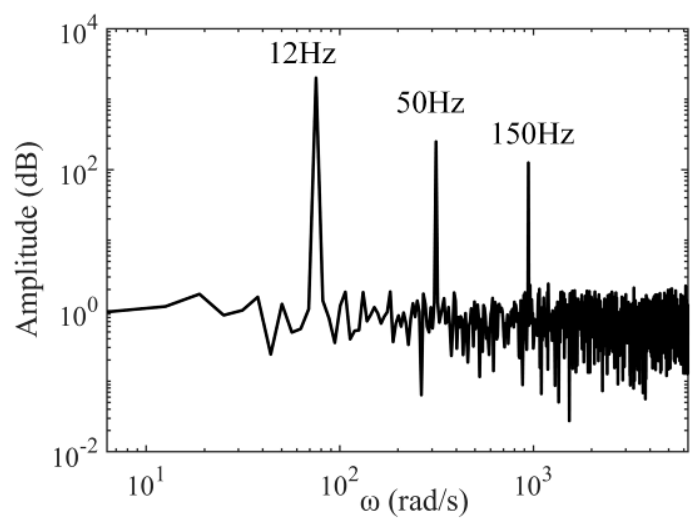

Fig. 3. Spectrum distribution of the synthetic signal 
term $\left\|\partial_{t}\left[\left(\delta(t)+\frac{j}{\pi t}\right) * u_{k}(t)\right] e^{-j \omega_{k} t}\right\|_{2}^{2}$ do not have adequate penalty, we can not expect the mode and center frequency could be precisely detected. When $\alpha$ is large, the constraint term $\left\|f(t)-\sum_{k=1}^{K} u_{k}(t)\right\|_{2}^{2}$ could not to be satisfied, we expect that the center frequencies $\omega_{k}$ and corresponding IMFs $u_{k}(t)$ might be estimated precisely under noisy and noise-free environments. However, the center frequencies would be convergence to the same frequency to lead mode redundant and missed due to the local optimization property of VMD [23]. Hence, value of $\alpha$ can be kept smaller (of the range of few hundreds) in order to capture signals containing wide range of frequencies; while for detection of frequency components of signal over smaller range, it can be kept larger (of the range of tens of thousands).

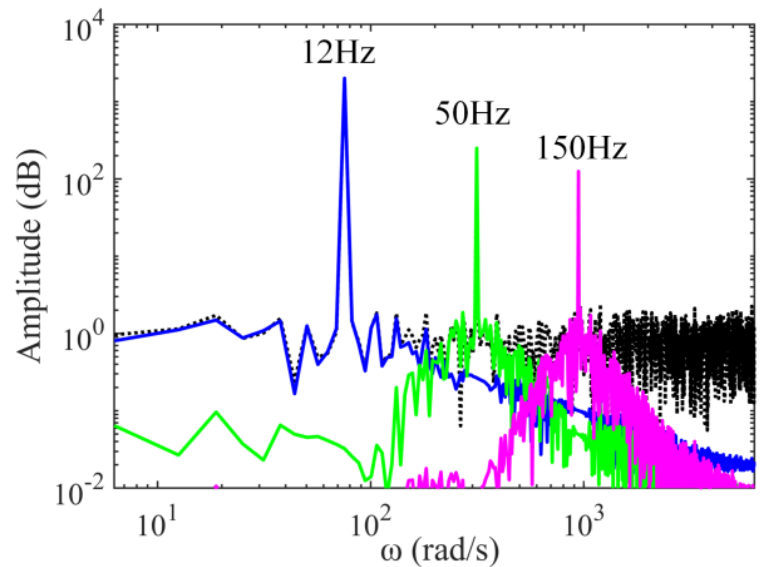

Fig. 4. The spectrum of the input signal (dashed line) and extracted

\section{SIMULATIONS AND EXPERIMENT STUDIES}

In this section, the proposed method was validated through synthetic and real-life signals. The performance of proposed method was evaluated in single-channel grid voltage harmonics separation and compared with EEMD-ICA and EMD-ICA methods.

\section{A. Synthetic Signal}

The synthetic signal was mixed with three frequencies recordings and Gaussian white noise into single channel mixture in MATLAB. Its formula is expressed as:

$$
\begin{aligned}
v(t)= & 2 \cos (2 \pi \times 12 t)+0.25 \cos (2 \pi \times 50 t)+ \\
& 0.125 \cos (2 \pi \times 150 t)+0.02 n(t)
\end{aligned}
$$

where the frequencies are $12 \mathrm{~Hz}, 50 \mathrm{~Hz}, 150 \mathrm{~Hz}$ respectively and $n(t)$ is Gaussian white noise. The sample frequency is set at $2000 \mathrm{~Hz}$ and the sample data is collected over $1 \mathrm{~s}$. Moreover, we made the amplitude of interharmonic is quadruple of fundamental voltage in order to evaluate the outstanding performance of the proposed method. A moderate level noise with Signal-to-Noise-Ratio(SNR) $=37 \mathrm{~dB}$ is used here. The waveform of mixture was illustrated in Fig.2, where Y-axis is the amplitude of mixture, $\mathrm{X}$-axis is sample time. Fig. 3 shows spectrum distribution of the signal, where Y-axis is the amplitude of frequency, $\mathrm{X}$-axis is the spectrum distribution which unit is rad/s. It can be seen from spectrum that there are $12 \mathrm{~Hz}$ interharmonic, $150 \mathrm{~Hz}$ harmonic, $50 \mathrm{~Hz}$ fundamental

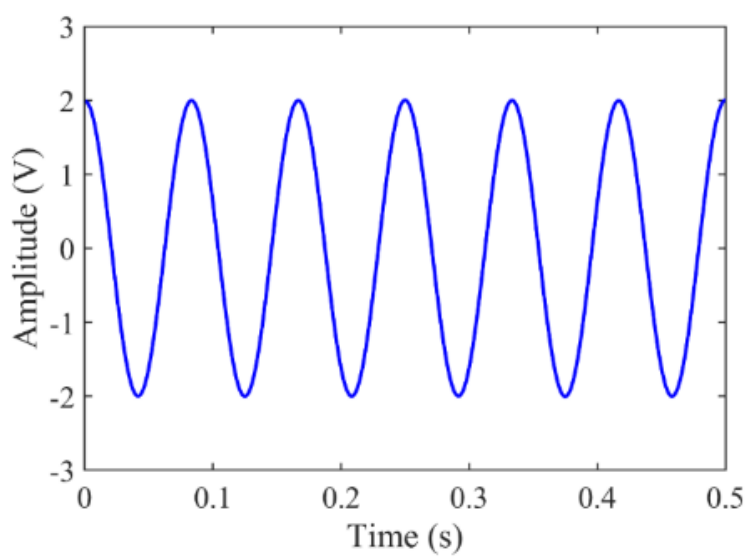

(a)

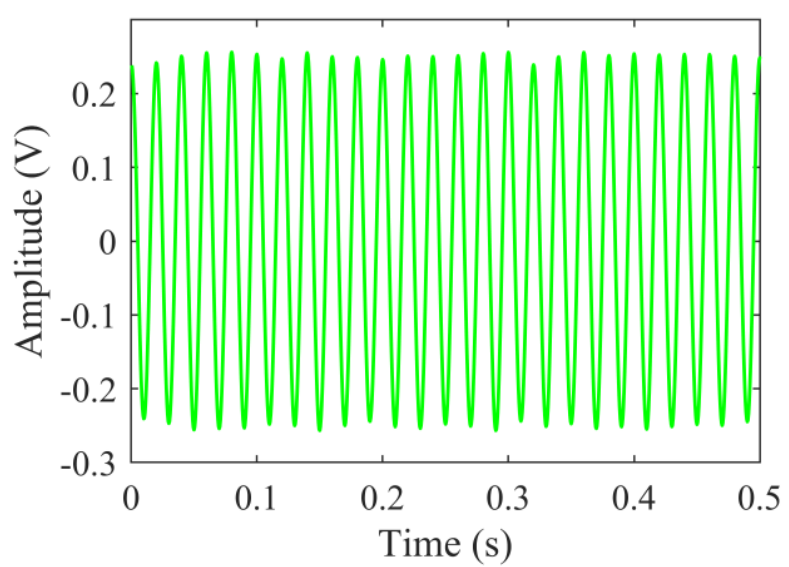

(b)

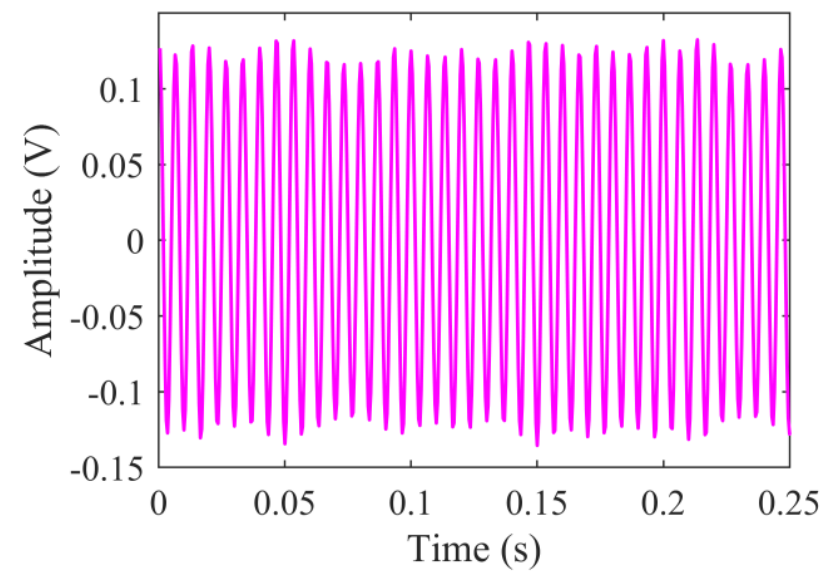

(c)

Fig. 5. The results of decomposition. (a) The $12 \mathrm{~Hz}$ mode extracted by VMD(solid), and the theoretical mode(dot) (b) The $50 \mathrm{~Hz}$ mode extracted by $\mathrm{VMD}$ (solid), and the theoretical mode(dot) (c) The $150 \mathrm{~Hz}$ mode extracted by VMD(solid), and the theoretical mode(dot)

component and Gaussian white noise in the single channel signal.

Next, we use the method to separate the synthetic signal. Considering both precise requirement and noisy level of voltage signal, $\alpha=2000$ was chosen. Moreover, $\mathrm{K}=3$ was set according to wave packages of FFT of voltage signal in the frequency domain. Fig. 4 shows the spectrum of the input signal and extracted modes. It evaluated that the spectrum of the three 
IMFs
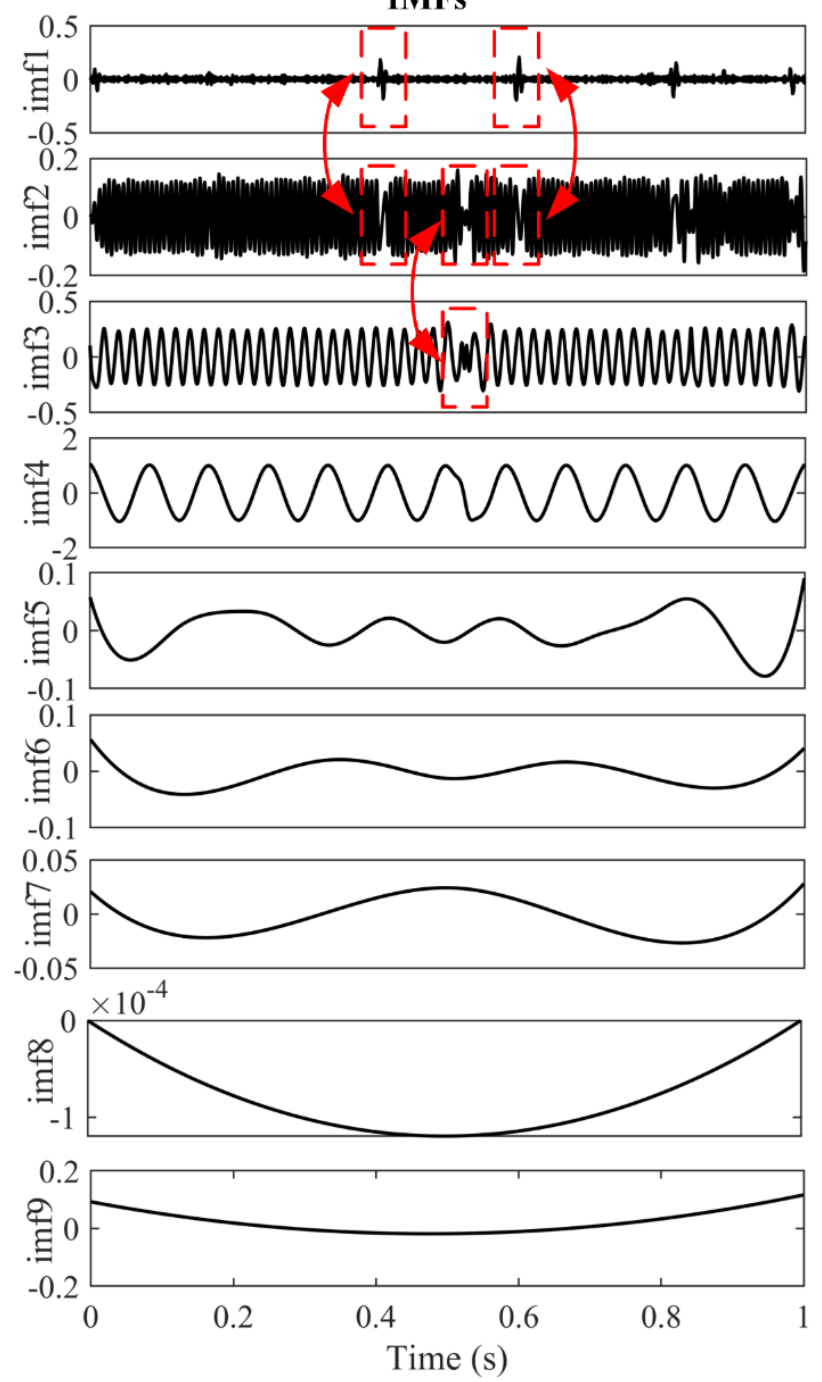

Fig. 6. The IMFs decomposed by the EMD method

frequencies were decomposed distinctly and had high degree of correlation with the theoretical modes. The modes of the signal which are recovered by VMD constitute a good partition of the original signal spectrum. It can be seen that each mode was clearly dominant around its respective center frequency. From Fig. 5, it can be seen that the three frequency parts of the single channel synthetic signal were well separated by means of the proposed method with both amplitude and frequency. The strongest with lowest frequency component was extracted almost flawlessly. The medium frequency and strength component was also recovered nicely. The lowest amplitude of the harmonics in the synthetic signal is close to noise. But the correlation coefficient $R$ of the component is 0.9982 from Eq. 11. Therefore, the third component was detected not as well as the other components, but also at acceptable level.

Furthermore, EMD-ICA and EEMD-ICA were also used to separate harmonics from the same synthetic signal. The EMD and EEMD methods were used respectively to decompose the single channel signal into finite number IMFs, which are illustrated in Fig. 6 and Fig. 7. According to EMD/EEMD algorithm [10], being different from VMD, the number of IMFs

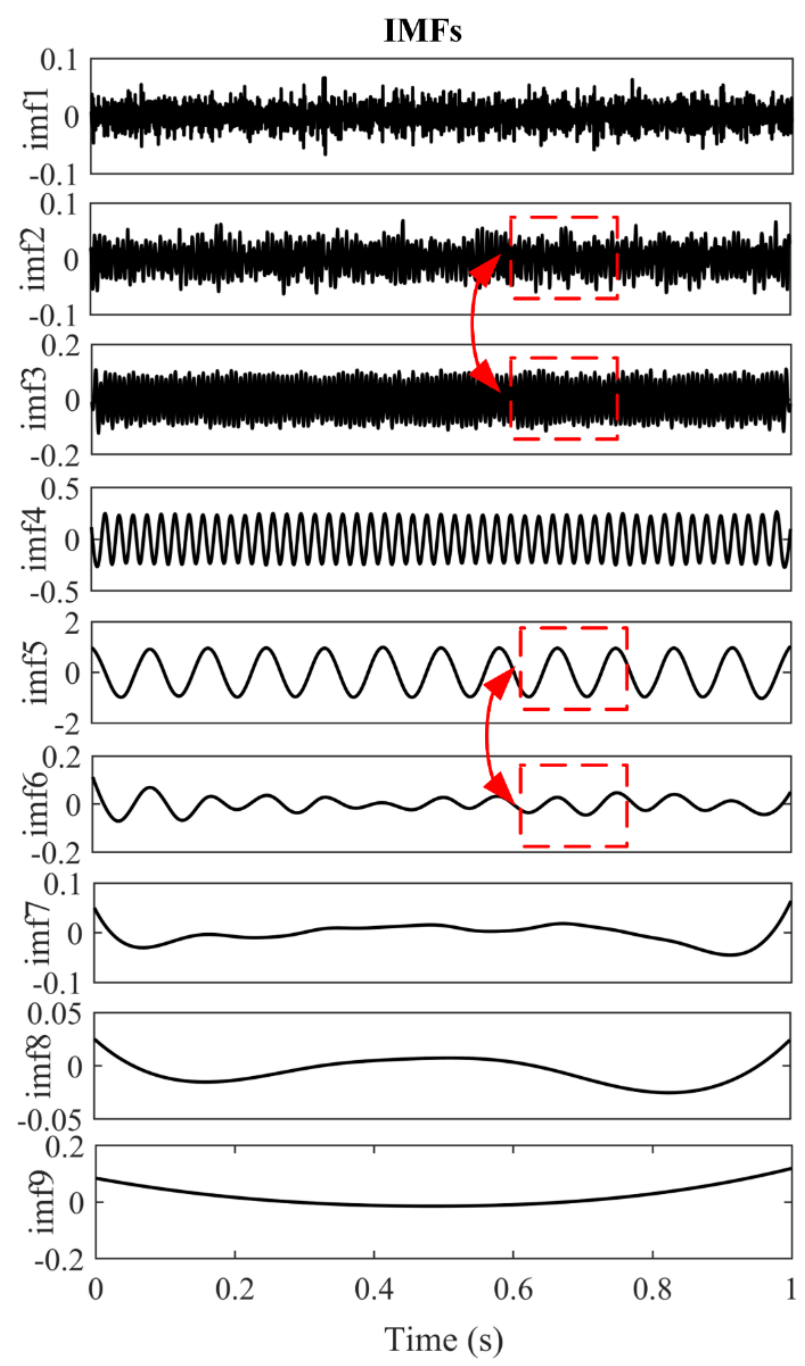

Fig. 7. The IMFs decomposed by the EEMD method

is not needed to choose artificially. All IMFs obtained through EMD/EEMD can be decomposed, when two conditions are satisfied [10]: 1) the number of extrema and zero crossings either be equal or differ at most by one; 2) the mean value of envelope defined by the local maxima and local minima is zero.

It can be seen from Fig. 6 that the intrinsic mode functions(IMFs) separated by EMD had mode mixing among each other (red rectangle). In Fig. 7, it can be seen that the IMFs separated from EEMD had less mixing modes than EMD method (also red rectangle). Although, EEMD made better effect on mode mixing, there were still some apparent limitations such as noise and sampling sensitivity etc. Therefore, a post-processing based on ICA is required. ICA could extract independent components from IMFs. The results extracted from EMD-ICA and EEMD-ICA methods are shown in Fig. 8, where $\mathrm{Y}$-axis is amplitude of mixture, $\mathrm{X}$-axis is number of samples.

After post-processing based on ICA, three components with different frequencies were extracted, which were illustrated as y1, y2 and y3. In fig. 8(a), some oscillatory transient (red rectangle) come up in the modes with $150 \mathrm{~Hz}$ and $50 \mathrm{~Hz}$ 
frequencies extracted by EMD-ICA method. The oscillatory transient can be eliminated by using EEMD-ICA method. Therefore, noise component was introduced into $12 \mathrm{~Hz}$ component in Fig. 8(b) (red rectangle). Besides, voltage amplitude was eliminated by EMD-ICA and EEMD-ICA methods (red circle) due to the limitations of the ICA algorithm.

Above all, it can be seen from Figs. 3-8 that the method proposed in this paper to separate harmonics and interharmonics from grid voltage based on VMD has better results than EMD-ICA and EEMD-ICA methods. Furthermore, this method reserved both frequency and amplitude of voltage

TABLE I

$R$ OF THREE METHODS WITH DIFFERENT FREQUENCIES

\begin{tabular}{cc|cc|cc}
\multicolumn{2}{c|}{ VMD } & \multicolumn{2}{c|}{ EEMD-ICA } & \multicolumn{2}{c}{ EMD-ICA } \\
\hline $\mathrm{f}(\mathrm{Hz})$ & $\mathrm{R}$ & $\mathrm{f}(\mathrm{Hz})$ & $\mathrm{R}$ & $\mathrm{f}(\mathrm{Hz})$ & $\mathrm{R}$ \\
12 & 1 & 12 & 0.9991 & 12 & 0.998 \\
50 & 0.9997 & 50 & 0.9861 & 50 & 0.9382 \\
150 & 0.9982 & 150 & 0.9898 & 150 & 0.906
\end{tabular}

TABLE II

THE COMPUTATIONAL TIME OF THREE METHODS

\begin{tabular}{c|c} 
Methods & Time $(\mathrm{s})$ \\
\hline VMD & 0.419 \\
EMD-ICA & 0.621 \\
EEMD-ICA & 0.798
\end{tabular}

otherwise than the methods based on ICA algorithm. Related coefficient $R$ was also introduced in this paper to evaluate the performance of the proposed method compared to EMD-ICA and EEMD-ICA methods quantitatively. The definition of $R$ is:

$$
R=\frac{\sum_{i=1}^{N}\left(X_{i}-\bar{X}\right)\left(Y_{i}-\bar{Y}\right)}{\sqrt{\sum_{i=1}^{N}\left(X_{i}-\bar{X}\right)^{2}} \sqrt{\sum_{i=1}^{N}\left(Y_{i}-\bar{Y}\right)^{2}}}
$$

where $\mathrm{N}$ is the number of sampling points, $X_{i}$ is original signal, $Y_{i}$ is the separation mode, and $\bar{X}, \bar{Y}$ are the mean of $X_{i}, Y_{i}$, respectively. Table I shows the $R$ of three methods with different frequencies.

Compared to EMD-ICA and EEMD-ICA, the proposed method based on VMD has the highest $R$ in all the components decomposed from the synthetic signal. In this paper, the Gaussian white noise is utilised to add to the synthetic signal. Tens of signals combining Gaussian white noise with different amplitudes are used in each method to calculate the relationship between R and SNR. It shows the good performance of the method quantitatively. Related coefficient $R$ changes with Signal-to-Noise-Ratio(SNR). The relation between $R$ and SNR with different methods is shown in Fig. 9. It shows that the proposed method has better performance than the other methods even in the low SNR condition. Moreover, the correlation coefficient $\mathrm{R}$ can also be used to indicate how good a set of important parameters are to achieve a VMD. As a rule of thumb, the parameters should be reconsidered if $R$ is less than 0.8 . This can be conducted by either increasing regularization factor $\alpha$ or reselect mode number $\mathrm{K}$.

Computational time plays an important role in the algorithm. Therefore, it is considered in this paper. Table II shows the cost time of three methods. It clearly found that the proposed method based on VMD used the least time compared the other ones.

\section{B. Experimental Signals}

In order to validate the proposed methodology under experimental operating conditions, this method was applied to $10 \mathrm{kV}$ power measured by voltage sensors (sample frequency is $3200 \mathrm{~Hz}$ ) in a wind farm. The objective of this experiment was to separate fundamental component, harmonics and interharmonics from single channel real-life voltage signal. The

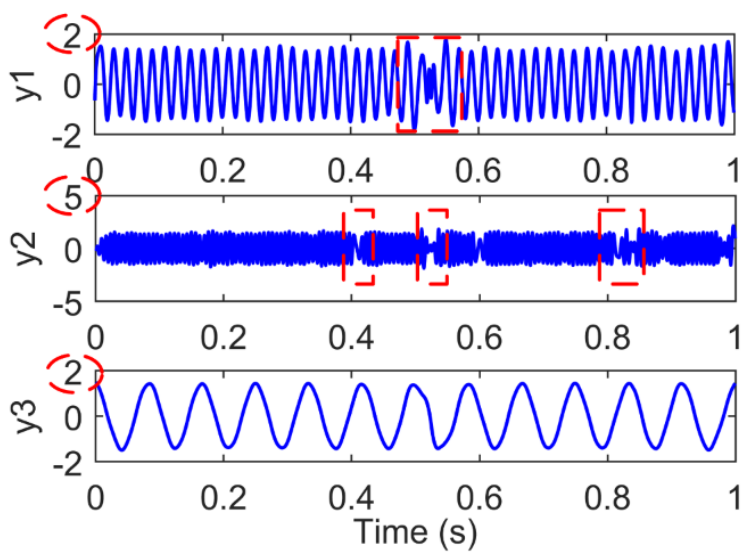

(a)

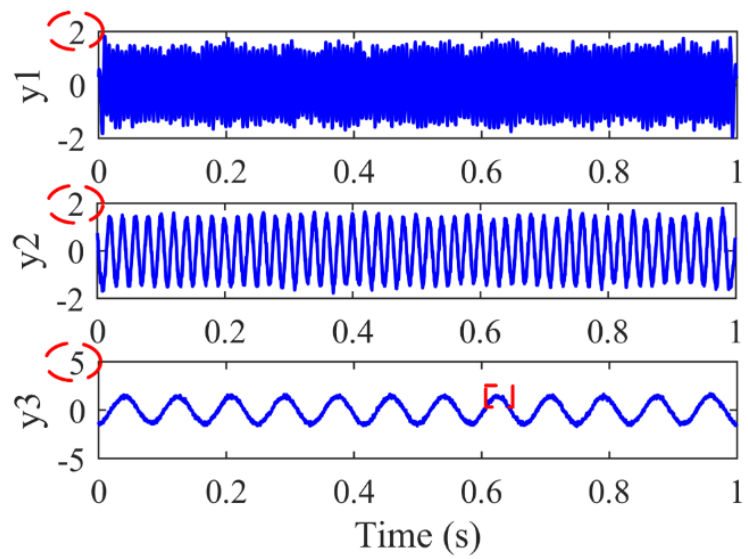

(b)

Fig. 8. The results extracted from EMD-ICA and EEMD-ICA methods. (a) EMD method (b) EEMD method

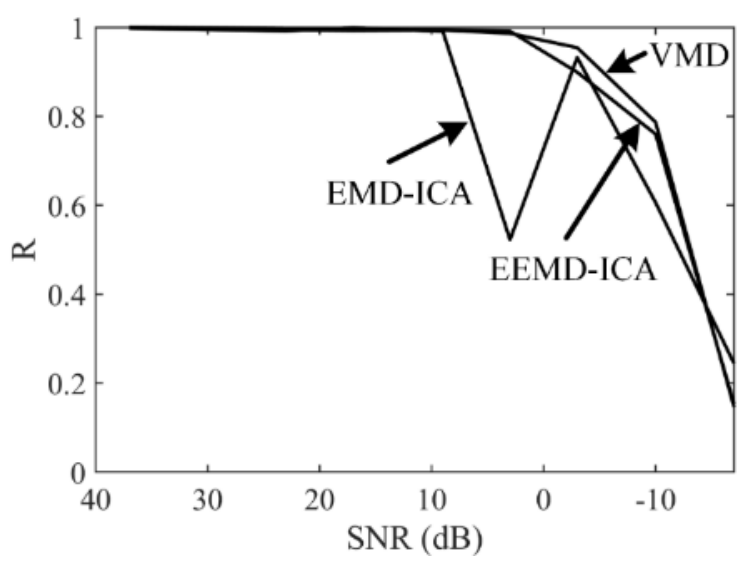

Fig. 9. The relation between $R$ and SNR with different method real-life signal and its spectrum was illustrated in Fig. 10. 
In this part, the number of modes $\mathrm{K}$ was chosen as 3 in order to separate the three components mentioned above. Fig.11 showed the decomposed modes and their spectrums using by the proposed method. The interharmonic $(10 \mathrm{~Hz})$, fundamental $(50 \mathrm{~Hz})$ component and high frequency oscillatory were separated clearly and reserved their amplitudes respectively. Using VMD to extract real-life signal showed that the modes recovered by VMD constitute a good partition of the original signal spectrum and each mode was clearly dominant around its respective center frequency. The modes also show nice separation into three distinct components of characteristic oscillations in time domain.

The EEMD-ICA and EMD-ICA were also used to extract the real-life signal as comparing to proposed method. The results can be seen in Fig.12. In Fig. 12(a), the fundamental component was not extracted clearly from the real-life signal. The high frequency oscillation was induced into the low frequency component (red circle), which is in accordance with the synthetic result. Also, it can be seen in Fig. 12(b) low frequency component was introduced in fundamental mode (red circle). Furthermore, the amplitude of real-life signal was eliminated by the EMD-ICA or EEMD-ICA methods.

The related coefficients of different methods were shown in Table III. The proposed method has the highest related coefficient among the three methods. Compared to the

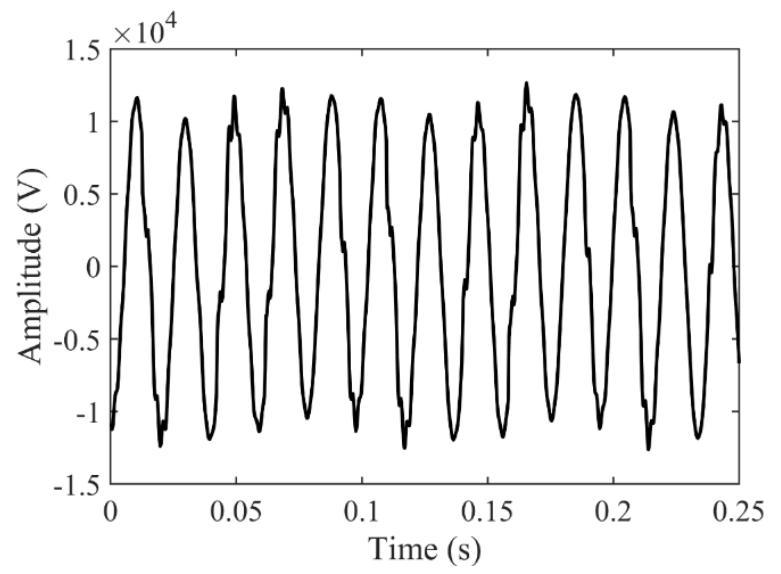

(a)

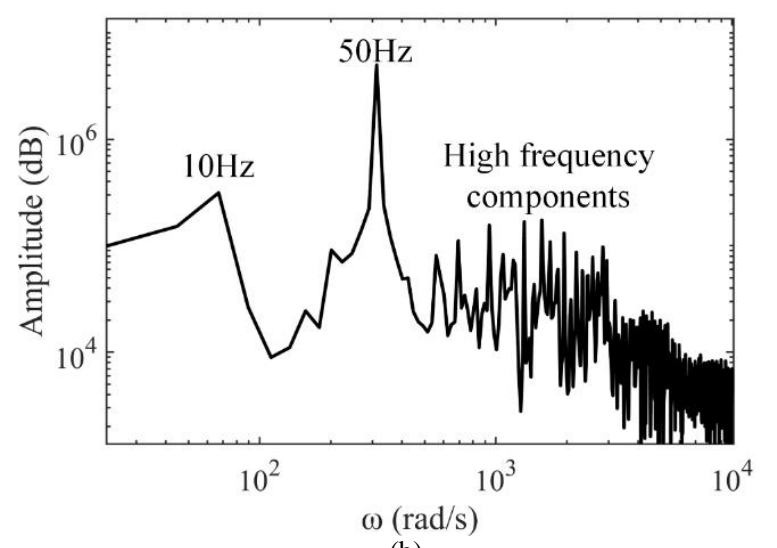

(b)

Fig. 10. The real-life signal and its spectrum. (a) the real-life signal; (b) the spectrum of the signal

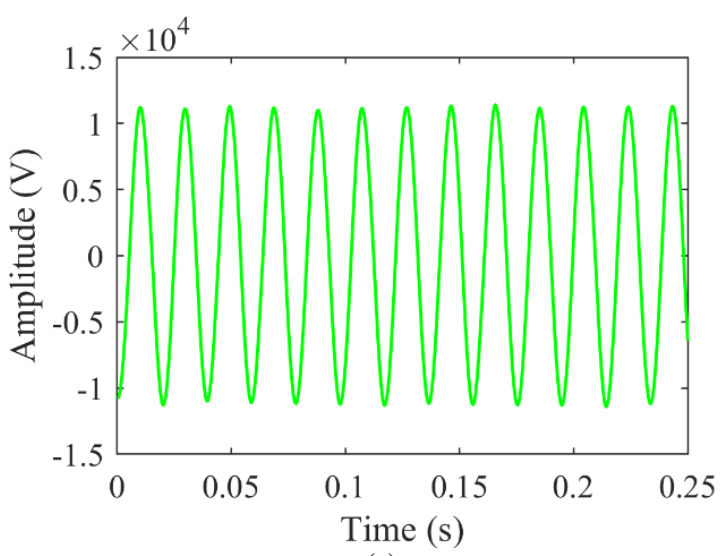

(a)

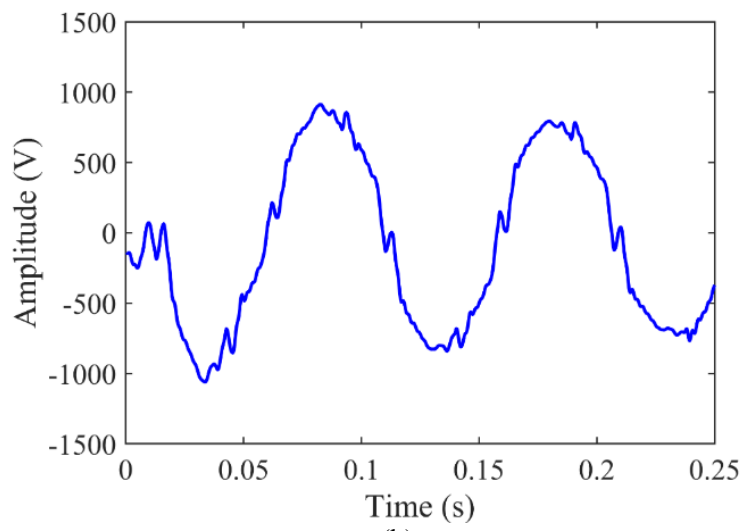

(b)

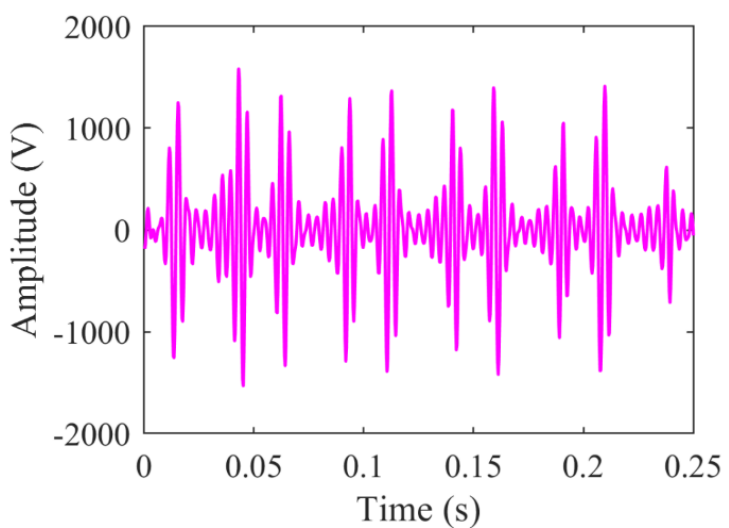

(c)

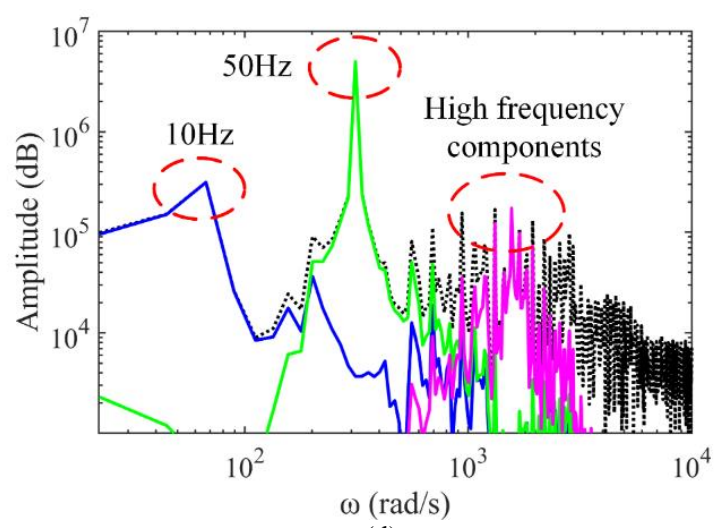

Fig. 11. The decomposed modes and their spectrums using by the proposed method. (a) fundamental component; (b) interharmonic component; (c) high frequency oscillatory; (d) spectrum distribution 
EMD/EEMD-ICA methods, the proposed VMD-based method to extract harmonics from grid voltage performs much better results, which has less spurious oscillations in the trend and higher related coefficients.

From the experiment results, it can be summarised as follows: 1) mode mixing and weak robust to noise in EMD-ICA affects drastically the correct decomposition of both synthetic and real-life voltage signal; 2) the proposed method can extract the components from synthetic and real-life signal exactly; 3 ) the proposed method has the highest related coefficient $R$ in all the components decomposed from the signal; 4) the proposed method requires much less time in decomposing the signal than EMD-ICA and EEMD-ICA; and 5) the proposed method is robust to the problem of mode-mixing. 7) voltage amplitude is reserved by the proposed method, while eliminated by EMDICA and EEMD-ICA.

\section{CONCLUSION}

In this paper, an approach based on VMD for separating harmonics and interharmonics from single channel voltage signal has been presented. The proposed method has been verified through synthetic and experimental signals of grid voltage, where the results indicate that the performance of the proposed method compared to EMD-ICA and EEMD-ICA is superior. After post-processing based on ICA, the amplitude of voltage signal is eliminated, while it is reserved by proposed

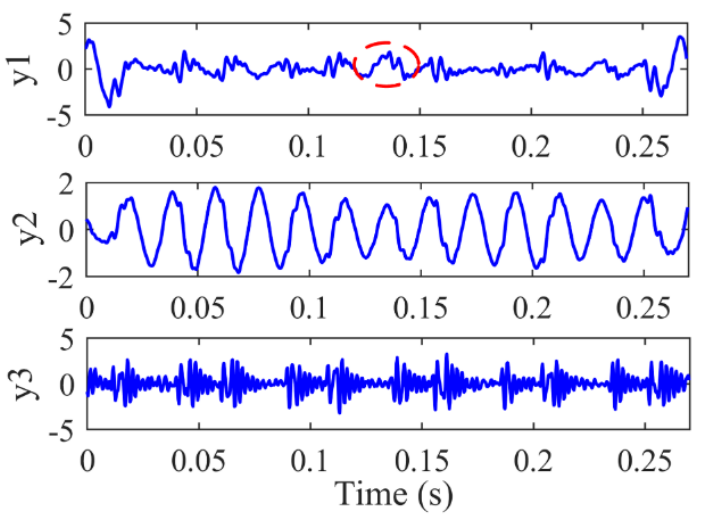

(a)
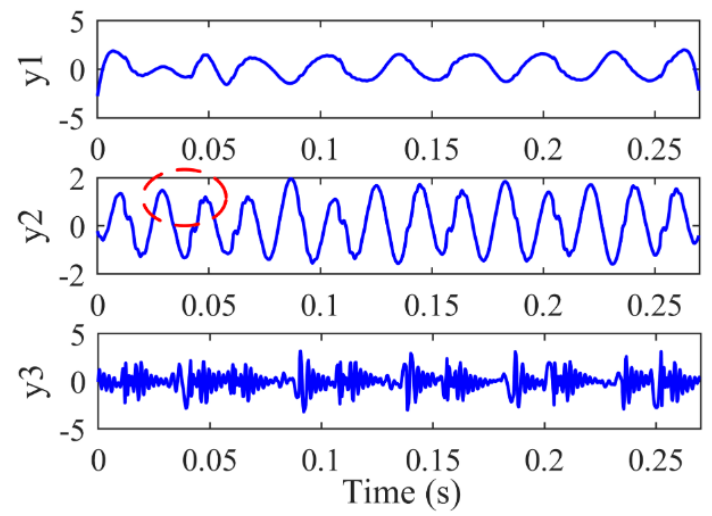

(b)

Fig. 12. Test results from EEMD-ICA and EMD-ICA based on real-life signals. (a) EEMD-ICA; (b) EMD-ICA
TABLE III

THE $R$ OF THREE METHODS WITH DIFFERENT FREQUENCIES
\begin{tabular}{c|crr} 
METHODS & VMD & EEMD-ICA & EMD-ICA \\
\hline R & 0.9992 & 0.8923 & 0.8865
\end{tabular}

method based on VMD. Moreover, the proposed method shows a greater robustness to the mode-mixing problem than EMDICA and EEMD-ICA. Furthermore, the method requires a minimal computational resource than conventional methods (EMD-ICA and EEMD-ICA). Therefore, large amounts of data as the case of real power systems can be analyzed online.

\section{FUNDING}

The authors gratefully acknowledge the support of the Foundation of Liaoning Province Education Administration (grant number L201609), the Doctoral Start-up Foundation of Liaoning Province (grant number 20170520191) and the Royal Society U.K.

\section{REFERENCES}

[1] T. Layadi, G. Champenois, and M. Mostefai, "Modeling and design optimization of an autonomous multisource system under a permanent power-supply constraint," IEEE Trans Sustain Energ., vol. 6, no. 3, pp. 872880, 2017.

[2] S. Alkaabi, V. Khadkikar, and H. Zeineldin, "Incorporating pv inverter control schemes for planning active distribution networks," IEEE Trans Sustain Energ., vol. 6, no. 4, pp. 1224-1233, 2015.

[3] S. Clegg and P. Mancarella, "Integrated modeling and assessment of the operational impact of olpower-to-gas (P2G) on electrical and gas transmission networks", IEEE Trans Sustain Energ., vol. 6, no. 4, pp. 1234$1244,2015$.

[4] A. Javadi, and A. Kamal, "A single phase active device for power quality improvement of electric field transportation," IEEE Trans Ind Electron, vol. 62, no. 5, pp. 3033-3041, 2015.

[5] G. Heydt et al., "Applications of the windowed FFT to electric power quality assessment," IEEE Trans Power Delivery, vol. 14, no. 4, pp. 1411-1416, 1999.

[6] D. Robertson, O. Camps, J. Mayer, and W. Gish, "Wavelets and electromagnetic power system transients," IEEE Trans Power Delivery, vol. 11, no. 2, pp. 1050-1058, 1996

[7] K. Cai, Z. Wang, G. Li, D. He, and J. Song, "Harmonic separation from grid voltage using ensemble empirical-mode decomposition and independent component analysis", INT T Electr Energy, vol. 27, no. 11, pp. e2405, 2017.

[8] M. E. Davies and C. J. James, "Source separation using single channel ICA," Signal Process, vol. 87, no. 8, pp. 1819-1832, 2007.

[9] X. Wu, X. Li, M. Kong, H. Feng, and H. Zhou, "Harmonic estimation and removal based on independent component analysis," Transactions of China Electrotechnical Society, vol. 18, no. 4, pp. 56-60, 2003.

[10] J. Yu, Y. Sun, and L. Jing, "Harmonic detection based on independent component analysis," Power System Protection \& Control, vol. 37, no. 8, pp. 14-18, 2009.

[11] L. Meng, C. Sun, and X. Wang, "A detection method for harmonic/interharmonic based on eigenvalue decomposition and fast independent component analysis," Automation of Electric Power Systems, vol. 36, no. 5, pp. 61-66, 2012.

[12] L. Meng, C. Sun, and B. Han, "Algorithm for inter-harmonic detection based on least square method and ICA,"Power System Protection \& Control, vol. 40, no. 11, pp. 76-81, 2012.

[13] B. Wang, N. Wang, Y. Jiang, Z. Cheng, and W. Bao, "Harmonic detection based on dynamic independent component analysis," Power System Protection \& Control, vol. 39, no. 2, pp. 40-44, 2011.

[14] D. Zheng, H. Chen, and Z. Yang, "Application of independent component analysis on harmonic source identification," Proceedings of the CSUEPSA, vol. 24, no. 4, pp. 139-144, 2012.

[15] N. E. Huang et al., "The empirical mode decomposition and the hilbert spectrum for nonlinear and non-stationary time series analysis," P Roy Soc 
A-Math Phy, vol. 454, no. 1971, 1998, pp. 903-995.

[16] Y. Lei, J. Lin, Z. He, and M. Zuo, "A review on empirical mode decomposition in fault diagnosis of rotating machinery," Mech Syst Signal $P R$, vol. 35 , no. 1-2, pp. 108-126, 2013.

[17] B. Mijovic, M. Vos, I. Gligorijevic, J. Taelman and S. Huffel, "Source separation from single-channel recordings by combining empirical-mode decomposition and independent component analysis," IEEE Trans Biomed Eng, vol. 57, no. 9, pp. 2188-2196, 2010.

[18] D. Camarena-Martinez et al., "Novel downsampling empirical mode decomposition approach for power quality analysis," IEEE Trans Ind Electron, vol. 63, no. 4, pp. 2369-2378, 2016.

[19] Y. Guo, S. Huang, and Y. Li, "Single-mixture source separation using dimensionality reduction of ensemble empiricalmode decomposition and independent component analysis," Circ Syst Signal Pr, vol. 31, no. 6, pp. 2047-2060, 2012.

[20] Y. Guo, S. Huang, Y. Li, and G. Naik, "Edge effect elimination in singlemixture blind source separation," Circ Syst Signal Pr, vol. 32, no. 5, pp. 2317-2334, 2013.

[21] J. Ma, Y. Li, C. Liu, and Y. Qiu, "A model-trajectory-based method for analyzing the time-varying oscillation characteristics of a fault system," Electr Mach Pow Syst, vol. 43, no. 7, pp. 781-791, 2015.

[22] C. Zhang, J. Yang, Y. Lei, and F. Ye, "Single channel blind source separation by combining slope ensemble empirical mode decomposition and independent component analysis," J Comput Inform Syst, vol. 8, no. 8, pp. 3117-3126, 2012.

[23] K. Dragomiretskiy and D. Zosso, "Variational mode decomposition," IEEE Trans Signal Proces, vol. 62, no. 3, pp. 531-544, 2014.

[24] W. Yang, Z. Peng, K. Wei, S. Pu, and W. Tian, "Superiorities of variational mode decomposition over empirical mode decomposition particularly in time-frequency feature extraction and wind turbine condition monitoring," IET Renew Power Gen, vol. 11, no. 4, pp. 443-452, 2017.

[25] A. Smruthy and M. Suchetha, "Real-time classification of healthy and apnea subjects using ECG signals with variational mode decomposition", IEEE Sens J, vol. 17, no. 10, pp. 3092-3099, 2017.

[26] Y. Xue, J. Cao, D. Wang, H. Du, and Y. Yao, "Application of the variational-mode decomposition for seismic time-frequency analysis," IEEE J-STARS, vol. 9, no. 8, pp. 3821-3831, 2017.

[27] Z. Gao, X. Wang, J. Lin, and Y. Liao, "Online evaluation of metal burn degrees based on acoustic emission and variational mode decomposition," Measurement, vol. 103, pp. 302-310, 2017.

[28] M. R. Hestenes, "Multiplier and gradient methods,"J. Optimiz. Theory Appl., vol. 4, no. 5, pp. 303-320, 1969.

[29] R. Rockafellar, "A dual approach to solving nonlinear programming problems by unconstrained optimization," Math. Program., vol. 5, no. 1, pp. 354-373, 1973.

[30] D. Bertsekas, "Constrained optimization and lagrange multiplier methods," in Computer Science and Applied Mathematics, Boston, MA, USA: Academic, vol. 1, 1982.

[31] G. Choi, H. Oh, and D. Kim, "Enhancement of variational mode decomposition with missing values," Signal Process, vol. 142, pp. 75-86, 2018. 